\title{
Analysis of Clinical, Pathological, and Treatment Factors in Gastric Cancer
}

\author{
Ömer PARLAK' ${ }^{1}$ Serap ULUSOY², Mehmet ÖZER²
}

'Department of General Surgery, Faculty of Medicine, Ankara Yıldırım Beyazıt University, Ankara, Turkey.

${ }^{2}$ Department of General Surgery, Ankara Ataturk Training and Research Hospital, Ankara, Turkey.

\section{ABSTRACT}

A retrospective study of 122 patients, who were operated between January 1989 and December 2001, was performed D1 dissection was performed in the operations. In this study, initiatives that do not leave macroscopic tumor behind were evaluated as curative resections. The patients who underwent total gastrectomy were mostly reconstructed with omega loop and Braun anastomosis. Among the patients, 81 (66.4\%) were male and 41 (33.6\%) female. The youngest patient was 26 and the oldest 82 years old. The average age of patients was 58.9 years. Fifty seven patients (46. 7\%) were treated with curative (R1) resection, while 20 (16.4\%) with palliative resection, 20 (16.4\%) with surgical bypass, 5 (4.2\%) with gastrostomy, 2 (1.6\%) with nutritional jejunostomy, and 18 (14.7\%) underwent only explorative laparotomy. Patients who were operated were identified as follows. 3 of the patients (2.4\%) were in Stage IA, 4 (3.2\%) in Stage IB, 23 (18.8\%) in Stage II, 11 (9.1\%) in Stage IIIA, 16 (13.2\%) in Stage IIIB, and $55(53.3 \%)$ in Stage IV. The average life expectancy in the survival analysis of all patients was $44( \pm 3)$ months and the 5-year survival rate was $42.5 \%$. Survival results were found to be consistent with western-sourced results. These results have led to the need of comparison of D1 and D2 dissections with a prospective randomized study.

Key words: Gastric cancer, D1-D2 dissection, survival analysis

\section{INTRODUCTION}

The incidence of gastric cancer varies in different countries around the world. Japan, Hungary, Poland, Chile, Malaysia, and Iceland are the countries that have higher rate of gastric cancer (1).

According to the data reported by Cancer Control Department of the Ministry of Health, in 1994, gastrointestinal system cancers in males have come second just after the respiratory system cancers in Turkey. Again according to these data, gastric cancer is ranked first among other gastrointestinal system cancers. Male/female ratio in the gastric cancer is approximately $3 / 2$ (2). This ratio is $2 / 1$ in the world. Early diagnosis and treatment are important in the gastric cancer. In Japan, where the gastric cancer is widespread, the early detection of gastric cancer through screening programs is now more prevalent.

The Japanese state that lymph node dissection in the surgical treatment of gastric cancer has a significant impact on survival. They also reported that the lymph node dissection ranks fourth among other prognostic factors (3).

The aim of this study is to analyze the survival of patients who underwent a surgical intervention in general surgery clinic with the diagnosis of gastric cancer and to compare the effects of various factors on survival. In addition to survival analysis, morphological and pathological data of gastric cancer were also assessed in the scope of this study. 


\section{MATERIALS AND METHOD}

A retrospective study of 122 patients, who were operated between January 1989 and December 2001, was performed. Files of patients, surgery reports, histopathological examination results, and track records were used as a basis for the research.

The purpose of this study was primarily to obtain the survival results of gastric cancer patients according to pathological stages. In pathological staging, mainly tumor, node, metastasis classification is used. Histopathological analysis is based on the World Health Organization classification. Computerized tomography (CT) and ultrasonography (USG) results that were performed preoperatively were compared with the operative findings. In this study, initiatives that do not leave macroscopic tumor behind were evaluated as curative (R1) resection.

\section{Statistical Analysis}

Survival analysis of patients was performed with the KaplanMeier test. In survival analysis, log rank was used in benchmark tests and $P<0.05$ was considered as statistically significant.

\section{Findings}

The 122 patients were operated with the diagnosis of gastric cancer, and 81 (66.4\%) of them were male while other 41 (33.6\%) patients were female. The youngest patient was 26 and the oldest 82 years old. The average age of patients was 58.9 (Table 1).

Fifty-seven patients (46.7\%) were smokers. The most common clinical finding was weight loss (74\%) (Table 2).

The tumor was detected most frequently localized in the antrum (52.5\%) (Table 3).

Patients who were operated were found to be as follows: 3 of them (2.4\%) were in Stage IA, 4 (3.2\%) in Stage IB, 23 (18.8\%) in Stage II, 11 (9.1\%) in Stage IIIA, 16 (13.2\%) in Stage IIIB, and 55 (53.3\%) in Stage IV (Table 4).

In this study group, initiatives that do not leave macroscopic
TABLE 1: Distribution of patients according to age groups

\begin{tabular}{lcc}
\hline Age Group & Number & Percentage (\%) \\
\hline 49 and under & 15 & 12,3 \\
$50-69$ years & 82 & 67,2 \\
70 and over & 25 & 20,5 \\
\hline
\end{tabular}

\begin{tabular}{lc}
\multicolumn{2}{l}{ TABLE 2: Clinical findings and incidence } \\
\hline Clinical Findings & Percent (\%) \\
\hline Weight loss & 74 \\
Epigastric pain & 70 \\
Anorexia & 30 \\
Melena & 20 \\
Bleeding & 5 \\
\hline
\end{tabular}

\begin{tabular}{lcc|}
\multicolumn{3}{l}{ TABLE 3: Location of primary tumor } \\
\hline Location of Primary Tumor & Number & Percentage (\%) \\
\hline Antrum & 63 & 51.6 \\
Corpus & 15 & 12.3 \\
Kardia & 9 & 7.4 \\
Fundus & 8 & 6.5 \\
Preplorik & 4 & 3.2 \\
Pyloric & 2 & 1.7 \\
Linitis plastica & 16 & 13.1 \\
Remnant & 5 & 4.2 \\
Total & 122 & 100 \\
\hline
\end{tabular}

tumor behind are evaluated as curative resections. Patients who underwent total gastrectomy were mostly (90\%) reconstructed with omega loops and Braun anastomosis (Table 5).

In 77 patients, the tumor was resectable (Table 6).

As shown in Table 7, 57 patients underwent curative resection. Of those, 15 had splenectomy, 1 underwent resection of the transverse colon, and 1 underwent metastasectomy from the fifth segment of the liver.

TABLE 4: Distribution of patients according to stages

\begin{tabular}{lccccccc}
\hline Stage & Stage IA & Stage IB & Stage II & Stage IIIA & Stage IIIB & Stage IV & Total \\
\hline Number of patients & 3 & 4 & 23 & 11 & 16 & 65 & 122 \\
Percentage (\%) & 2,4 & 3,2 & 18,8 & 9,1 & 13,2 & 53,3 & 100 \\
\hline
\end{tabular}




\begin{tabular}{|c|c|c|}
\hline Interventions & $\begin{array}{c}\text { Number of } \\
\text { Patients }\end{array}$ & $\begin{array}{c}\text { Percentage } \\
(\%)\end{array}$ \\
\hline Curative resection & 57 & 46.7 \\
\hline Palliative resection & 20 & 16.4 \\
\hline Surgical bypass & 20 & 16.4 \\
\hline Gastrostomy & 5 & 4.2 \\
\hline Jejunostomy & 2 & 1.6 \\
\hline Exploratris laparotomy & 18 & 14.7 \\
\hline Total & 122 & 100 \\
\hline
\end{tabular}

TABLE 6: Types of operations performed for resectable cases

\begin{tabular}{lcc}
\hline Resectable & $\begin{array}{c}\text { Number of } \\
\text { Patients }\end{array}$ & $\begin{array}{c}\text { Percentage } \\
\text { Cases }\end{array}$ \\
\hline Radical distal gastrectomy & 52 & 67.5 \\
Partial proximal gastrectomy & 5 & 6.5 \\
Total gastrectomy & 20 & 26 \\
\hline
\end{tabular}

\begin{tabular}{lcc}
$\begin{array}{l}\text { TABLE 7: Types of operations in cases where curative resection } \\
\text { was performed }\end{array}$ & Number of & $\begin{array}{c}\text { Percentage } \\
\text { Curative }\end{array}$ \\
Resections & 42 & 73,7 \\
\hline Radical distal gastrectomy & 12 & 21.1 \\
Total gastrectomy & 3 & 5.2 \\
Partial proximal gastrectomy & & \\
\hline
\end{tabular}

All the patients who underwent palliative resection were Stage IV patients. Ten of 20 patients treated with palliative interventions were advised radical distal gastrectomy. Total gastrectomy was performed in 8 patients and partial proximal gastrectomy in 2 patients.

In 16 cases (13\%), morbidity was developed in the first 1 month after the surgery (Table 8).

The number of early mortality in this study is 7 (5.6\%) (Table 9).

In the survival analysis performed in this study, the average life expectancy was $23( \pm 2)$ months and median was 15 months. The 5 -year survival rate was $18.5 \%$. The average survival time of 57 patients treated with curative resection was $44( \pm 3)$ months and median was 48 months. The 5 -year survival rate was $42.5 \%$. Among the 20 patients treated with palliative resection, average life expectancy was $15( \pm 2)$ months and median was 13 months.
TABLE 8: Postoperative morbidities

\begin{tabular}{lcc}
$\begin{array}{l}\text { Postoperative } \\
\text { Morbidity }\end{array}$ & $\begin{array}{c}\text { Number of } \\
\text { Patients }\end{array}$ & $\begin{array}{c}\text { Percent } \\
(\%)\end{array}$ \\
\hline Anastomosis leakage & 5 & 4.1 \\
Evisceration & 4 & 3.2 \\
Wound infection & 3 & 2.5 \\
Deep vein thrombosis & 2 & 1.6 \\
Upper gastrointestinal & 1 & 0.8 \\
bleeding & & \\
Lung infection & 1 & 0.8 \\
\hline
\end{tabular}

\begin{tabular}{lcc} 
TABLE 9: Mortalities seen in early stages & \\
\hline Early Mortality & Number of Patients & Percent (\%) \\
\hline Sepsis & 2 & 1.6 \\
Evisceration and ARDS & 2 & 1.6 \\
Renal insufficiency & 2 & 1.6 \\
Cerebral hemorrhage & 1 & 0.8 \\
\hline
\end{tabular}

There was no 5 -year survival, and 2-year survival rate was 30\%. When 77 patients were treated with either palliative or curative resection evaluated together, the average life expectancy was 33 $( \pm 3)$ months and median was 22 months. The 5 -year survival rate was 22\% (Table 10).

When the patients were considered according to stages, in Stage I, the average survival was $59( \pm 3)$ months and median was 61 months. The 5 -year survival was $71 \%$. In Stage II, the average survival was $47( \pm 3)$ months, median was 51 months, and 5-year survival was 40\%. In Stage IIIA, the average survival was $38( \pm 4)$ months, median was 30 months, and 5 -year survival was $27 \%$. In Stage IIIB, the average survival was 20 ( \pm 2 ) months, median survival was 20 months, and 2-year survival was 68\%. In Stage IV, the average survival was $9( \pm 1)$ months, median was 6 months, and 2-year survival was 10\% (Table 11).

The average survival time was $5.6( \pm 1)$ months in 18 patients (14.7\%) who were considered inoperable and whose surgeries were terminated following the biopsy.

Histopathological examination revealed that 89 patients had adenocarcinoma, 10 of these patients were well differentiated, 20 were moderately, and 10 poorly differentiated; 11 of the patients had mucinous adenocarcinoma as well. The signet ring cell carcinoma was found in 6 patients, while differentiation of 27 patients was unknown. 


\begin{tabular}{|c|c|c|c|c|c|}
\hline Resections & Number of Patients & Average Survival (months) & 5-Year Survival (\%) & 2-Year Survival (\%) & Median \\
\hline Curative & 57 & 44 & 42.5 & - & 48 \\
\hline Palliative & 20 & 15 & - & 30 & 13 \\
\hline Curative + Palliative & 77 & 33 & 22 & - & 22 \\
\hline
\end{tabular}

\begin{tabular}{|c|c|c|c|c|c|}
\hline Stages & Average Survival (month) & 2-Year Survival (\%) & 5-Year Survival (\%) & Median & Number of patients \\
\hline Stage II & 59 & - & 71 & 61 & 7 \\
\hline Stage II & 47 & - & 40 & 51 & 23 \\
\hline Stage IIIA & 38 & - & 27 & 30 & 11 \\
\hline Stage IIIB & 20 & 68 & - & 20 & 16 \\
\hline Stage IV & 9 & 10 & - & 6 & 65 \\
\hline
\end{tabular}

Preoperative USG results in 95\% patients (118) were similar to the findings that were detected instantly in the operation. In 6 patients (5\%), negative results were found. Preoperative computed tomography was performed in 27 patients at the same time and false negative results were detected in 2 patients (7\%).

While any elevation was not detected in the tumor makers of patients with Stage I or II tumors, in patients with Stages III and IV tumors, carcinoembryonic antigen (CEA) and Carbohydrate Antigen 19-9 (CA 19-9) values were found, respectively, 55\%$45 \%$ normal and $45 \%-55 \%$ high.

\section{DISCUSSION}

According to the data reported by Cancer Control Department of the Ministry of Health, in 1994, gastrointestinal system cancer in males has come second after the respiratory system cancer in our country. Again, according to these data, gastric cancer was ranked first among other gastrointestinal system cancers. Male/ female ratio in gastric cancer was approximately 3/2 (2).

The depth of the tumor invasion, lymph node involvement, and existence of distant metastases were considered to be the most important prognostic factors in gastric cancer. In addition to these, adequate lymph node dissection was considered as the fourth important prognostic factor (3).

In the United States and the United Kingdom, D1 dissection is performed in cases where curative surgical can be performed (4). In our clinic, D1 dissection is preferred mostly.
The Japanese Gastric Cancer Research Group enacted standardized lymph node regions pursuant to the settlement of gastric cancer. However, for the survival of the patients, splenectomy should be avoided as much as possible as it causes postoperative complications (5). In the present study group, splenectomy was also performed in 15 (26\%) of 77 patients treated with curative and palliative reaction.

In retrospective studies, the 5-year survival was found as $27 \%$ in Stage II patients treated with D1 resection, whereas it was 55\% in the patients treated with D2 resection. In the same study, it was found as 25\% in Stage IIIA treated with D1 resection, and 38\% with D2 resection (6). In a study that compared the limited $(D 1, D 1,5)$ and large $(D 2, D 2,5)$ resections that were performed in proximal gastric cancer cases, the 5-year survival rate was detected in 21\% in limited resection, and 37\% in large resections; the average survival was 18 months in limited resection and 34 months in large resection. These results were found to be statistically significant (7). In this study, when all the patients were evaluated, the 5-year survival was found as $18.5 \%$.

In the prospective randomized studies conducted in Europe, there was no significant difference in the survival between D1-D2 and D1-D3 resections. In a study conducted in the Netherlands, 711 patients treated with D1 and D2 resections were compared. In the D2 resection group, complication occurred in $43 \%$ of the patients, and hospital mortality was 10\%; in the D1 resection group, complications occurred in $25 \%$ of the patients, and hospital mortality was 4\%. In the D2 resection group, distal 
pancreatectomy (D1: 3\%, D2: 30\%) and splenectomy (D1: 11\%, D2: 38\%) rates were significantly higher. In a group that developed complications, splenectomy and extensive lymph node dissection were identified as the most important risk factors. The age and gender was found to be the most important risk factors in mortality, and these risk factors were followed by wide lymph node dissection with splenectomy (8). A similar study was conducted in the United Kingdom. In this study, morbidity was $28 \%$ in the D1 resection group and $46 \%$ in the D2 resection group; the mortality rate was $6.5 \%$ in the D1 resection group and $13 \%$ in the $\mathrm{D} 2$ resection group (9). Although both studies failed to provide the 5-year survival, in the study conducted in the Netherland the 3-year survival rate was 60\% in D1 resection, while 55\% in D2 resection. A study conducted in Italy with 267 patients and published in 2014, no difference was found between the D1 and D2 groups in the 5-year survival rate, and this study suggested that D2 lymphadenectomy would be a better choice in the subgroups composed of patients with advanced disease and lymph node metastasis (10). Another meta-analysis of 1599 patients was published in 2015 covering the period between 1985 and 2014 years; similar results for 5-year survival were found in D1 and D2 resections and it was stated that D2 lymphadenectomy should be considered in subgroups called patients with advanced disease and lymph node metastasis (11).

In 70 years or older patients, although there was no difference in mortality related to the gastric cancer surgery, increase in morbidity was observed. Besides, when four groups formed with 10 years starting from age 50, there was no significant specific difference between postoperative complication rates. However, in patients who were 80 years or older, more limited resection and lymph node dissection has been proposed. It was stated that such a surgery does not have detrimental effect on the prognosis of patients in this age group (12). In this study, two patients died from renal failure; one was 64, while the other was 70 years. No differences were detected between the groups in terms of morbidity.

Advanced gastric cancer rate is higher in Western countries. Stage IV gastric cancer was 30\% in Western publications, and it has been reported that this ratio was $21 \%$ in Japan (13). Stage IV rate was $53.3 \%$ in this study. The reason for this is that the patients were late in applying to hospitals.
The 5-year survival rate in early gastric cancer was over 95\% while this rate was under 10\% in advanced gastric cancer (14). It has been reported that this rate can be increased to 20\%-30\%, if curative resection could be done in advanced gastric cancer (15). In the present study, when all Stage IV patients were evaluated, the average life expectancy was 9 months, while it was 15 months in cases with palliative resection. The 5-year survival was not detected in both the groups.

The treatment of gastric cancers that cause liver metastases remains controversial. It is known that liver resection performed in the colon tumors that cause liver metastases does not affect the survival. In a study made by the Japanese, synchronous metastasis liver resection was suggested in the absence of serous invasion of tumor. If there is no serous invasion in primary tumor, liver resection is suggested in the metachronous liver metastases that do not have venous or lymphatic invasion (16). In this study, only one patient underwent metastasectomy.

In the diagnosis, prognosis, and follow-up of tumors, tumor markers were utilized. Despite the use of multiple tumor markers in gastric tumors, these markers were far from perfect. CEA and CA 19-9 values were positive in gastric cancer patients at rates of, respectively, $24 \%-33 \%$ and $28 \%-46 \%(17,18)$. That tumor marker is higher than normal level is associated with tumor's making metastasis and its depth and size in the stomach wall (19). In this study, no elevation in CEA and CA19-9 values was detected in Stages I and II patients.

Preoperative staging is important in the treatment planning of gastric cancer patients. CT and USG are the most widely used radiological diagnostic methods for this purpose. Preoperative radiological evaluation of gastric cancer patients were compared with surgical and pathological findings. In a meta-analysis published in 2012, it was stated that in detecting lymph node staging (N staging), magnetic resonance has been reported to be $75.0 \% \pm 9.3 \%$ specific, CT $78.3 \% \pm 2.5 \%$, and ultrasound $78.8 \% \pm 13.9 \%$ (20). In this study, it was found that USG has 95\% certainty and CT has 93\% certainty in detecting liver metastases. Preoperative MRI was not used.

In the analysis conducted in Japan, among 1150 patients operated with diagnosis of gastric cancer between the years 1965 and 1974, tumor in gastric antrum was detected in 46\%, tumor in corpus in $27.2 \%$, and cardia localized tumor in $26.8 \%$ 
of patients. However, between 1975 and 1985, these rates had changed as follows: Antrum in 37\%, corpus in 31.6\%, and cardia in $31.4 \%$ of patients (21). As for the present study, these rates were found as $51.6 \%$ for antrum, $12.3 \%$ for corpus, and $4 \%$ for cardia-localized gastric tumors. In addition, 13\% linitis plastica and $4 \%$ remnant gastric cancer were detected in $4 \%$ of the cases.

\section{RESULTS}

Today, the treatment of gastric cancer is only possible with surgery. Especially in the early stages of gastric cancer, survival rates of over $80 \%$ can be achieved with radical surgical resection.

Especially mass screening method performed with endoscopy has increased the rate of success in countries such as Japan where gastric cancer is common more than 10 times; it is not efficient in the western-origin series, including our country. The Japan series suggesting and applying a wider lymph node dissection to ensure local control for gastric cancer are superior to westernorigin series, especially for Stages II and IIIA.

However, in the recent prospective randomized studies in the west, no significant difference between D1 and D2 dissection was found. This suggests the literature battle between the world's two regions will be continued.

To create more clarified results, it is required to wait for a longer and wider series of results and perhaps to create joint working protocols. This study conducted with D1 dissection revealed the same survival results with the western series.

In this study, survival results of Stages IIIB and IV patients, which composed the majority, show that there is no chance of treatment as long as tumors are not detected in earlier stages. Gastric cancer, in fact, is more common in our country than it is thought; success rate of the surgical treatment of gastric cancer will increase with more common endoscopic controls and with the implementation of different methods, such as chromoendoscopy.

\section{REFERENCES}

1. Whelen S, Parkin D, Masuyer E, Patterns of cancer incidence in five continents IARC. Sci Publ no 102. Lyon, France: International Agency for Research on Cancer, 1990

2. T. C. Sağlık Bakanlığı Kanser Savaş Daire Başkanlığı yayın no: 552 Ankara, 1994,59.

3. Maruyama K, Sasako M, Kinoshita T. Et al. Optimum resection with lymph node dissection for gastric cancer in: Wanebo $\mathrm{H}$, editor. Surgery for gastrointestinal cancer. Philadelphia: Lippincott-Raven, 1997:319-25
4. Cuschieri A. Gastrectomy for Gastric Cancer: Definitions and Objectives Br. J. Surg. 1986; 73: 513-14

5. Otsuji E, Yamaguchi T, Sawai K. Et al. End results of simultaneous splenectomy in patients undergoing total gastrectomy for gastric carcinoma. Surgery 1996;120:40-44

6. Noguchi M, Miyazaki I. Prognostic significance and surgical management of lymph node metastasis in gastric cancer. Br. J. Surg. 1996, 83: 156-61

7. Volpe MC, Driscoll DL, Miloro SM, et al. Survival benefit of extended D2 resection for proximal gastric cancer. J. Surg. Onco. 1997; 64: 231-36

8. Sasako M. Risk factors for surgical treatment in the Dutch gastric cancer trial. Br. J. Surg. 1997, 84: 1567-71

9. Cuschieri A, Joypaul V, Fayers B, et al. Postoperative morbidity and mortality after D1 and D2 resections for gastric cancer. The Lancet. 1996, 347: 995-99

10. M. Degiuli, M. Sasako et al. Randomized clinical trial comparing survival after D1 or D2 gastrectomy for gastric cancer. Br. J. Surg. 2014, 101: 23-31

11. Elsedfy A, Dixon M, Seevaratnam R. et al. Personelized surgery for gastric adenocarcinoma: a meta-analysis of D1 versus D2 lymphadenectomy. Annal of surgical oncology 2015, 22, 6: 1820-7

12. Tsujitani S, Katano K, Oka A, Ikeguchi M, Maeta M, Kaibara N. Limited operation for gastric cancer in the elderly. Br. J. Surg. 1996, 6: 836-9

13. Kodera Y,Yamamura $Y$, Torii A. et al. Postoperative staging of gastric carcinoma. A comparison between the UICC Stage Classification and the 12th edition of the Japanese General Rules for Gastric Cancer Study. Scand. J. Gastroenterol. 1996; 31: 476-80

14. Breaux JR. Bringaze W, Vahappuis C, et al. Adenocarcinoma of the stomach: A review of 35 years and 1710 cases. World J Surg. 1990; 14: 580-6

15. Shiu MH, Perrotti M, Brennan MF. Adenocarcinoma of the stomach: A multivariate analysis of clinical, pathologic and treatment factors. Hepatogastroenterology 1989; 36:7-12

16. Ochiai T, Sasako M, Mizuno $S$, et al. Hepatic resection for metastatic tumours from gastric cancer: Analysis of prognostic factors. Br. J. Surg. 1994; 81: 1175-8

17. Nakajima K, Ochiai $T$, Suzuki $T$, et al. İmpact of preoperative serum CEA, CA19-9 and AFP levels in gastric cancer patients. Tumour Biol. 1998; 19:464-9.

18. Filella X, Fuster J, Molina R, et al. TAG 72, CA19-9, CEA as tumour markers in gastric cancer. Acta Oncol. 1994;33:757-51

19. Kodera $Y$, Yamamura $Y$, Torii $A$, et al. The prognostic value of preoperative serum levels CEA and CA19-9 in patient with gastric cancer. Am. J. Gastroenterol. 1996;91:49-53

20. Rajini S, Roberta C, Caitlin M, Laercio L, Alyson M, Rinku S, Calvin $L$, Lawrence $P$, Natalie C. How useful is preoperative imaging for tumor, node, metastasis(TNM) staging of gastric cancer? A meta-analysis. Gastric Cancer 2012;15:3-18

21. Korenaga $D$, Moriguchi $S$, Orita $H$, Kakeji $Y$, Haraguchi $M$, Maehara $Y$, Sugimachi K. Trends in survival rates in Japanese patients with advanced carcinoma of the stomach. Surgery, Gynecology \& Obstetrics 1992;174(5):387-93. 